\title{
Precoder Design for OSTBC Based AF MIMO Relay System With Channel Uncertainty
}

\author{
Batu K. Chalise, Senior Member, IEEE, Yimin D. Zhang, Senior Member, IEEE, and Moeness G. Amin, Fellow, IEEE
}

\begin{abstract}
The source and relay precoders are jointly optimized for an amplify-and-forward multiple-input multiple-output relay system. Both the source and relay nodes employ orthogonal spacetime block codes (OSTBC), and have imperfect channel state information of the source-relay and relay-destination channels, respectively. Using the worst-case robust design approach, we show that the problem of maximizing the minimum signal-to-noise ratio at the destination can be exactly reformulated as a convex optimization problem. Further, we provide an approximate semi-analytical approach which significantly reduces the computational cost of solving the convex problem. Numerical results show that this approximation is accurate and the proposed design outperforms OSTBC with eigen beamforming, OSTBC with equal power allocation, and currently available worst-case robust beamforming design without OSTBC.
\end{abstract}

Index Terms-Imperfect CSI, MIMO relay, OSTBC precoding.

\section{INTRODUCTION}

$\mathbf{R}$ ECENTLY, designs that provide robustness against imperfect channel state information (CSI) in multiple-input multiple-output (MIMO) relay systems have garnered significant interest. In [1] uncertainties of the source-relay (S-R) and relay-destination (R-D) channels are described using a stochastic model and the corresponding average mean-square error (MSE) is minimized. Assuming that the S-R channel is perfectly known at the source, and only the mean and covariance of the R-D channel are available, the joint optimal source and relay precoder matrices are obtained in [2] with a criterion to maximize the upper bound of the ergodic capacity. However, stochastic approach can only guarantee desirable average performance. Alternatively, uncertainty can be described using norm bounded error models which result into robust designs based on the worst-case approach [3]. In [4], a robust design of relay precoder based on the worst-case design method is proposed. However, this work considers only the optimization of the relay precoder and, thus, imperfectness is only present in the R-D channel. To the best of our knowledge, the joint optimization of robust source and relay precoders

Manuscript received May 22, 2012; accepted June 03, 2012. Date of publication June 15, 2012; date of current version June 29, 2012. This work was supported in part by Defense Advanced Research Projects Agency (DARPA) under Contract HR0011-10-1-0062. The content of the information does not necessarily reflect the position or the policy of the Government, and no official endorsement should be inferred. Approved for Public Release, Distribution Unlimited. The associate editor coordinating the review of this manuscript and approving it for publication was Prof. Yong Liang Guan.

The authors are with the Center for Advanced Communications, Villanova University, Villanova, PA 19087 USA (e-mail: batu.chalise@villanova.edu; yimin.zhang@villanova.edu; moeness.amin@villanova.edu).

Color versions of one or more of the figures in this paper are available online at http://ieeexplore.ieee.org.

Digital Object Identifier 10.1109/LSP.2012.2204979 based on the worst-case design approach is an open topic, even for a single-stream data transmission, and has not yet been considered. A tractable solution for this open optimization problem is rather difficult. This is mainly due to the fact that the performance metrics such as sum MSE or signal-to-noise ratio (SNR) in the presence of errors are not only nonconvex functions of precoders but also consist of cross terms between S-R and R-D channel estimation errors and their second-order terms. The aforementioned optimization problem can be approximately solved using the robust approach of [5]. However, this design becomes conservative and further assumes that the errors are sufficiently small (w.r.t. estimated channels) so that their second-order terms are negligible.

In view of the existing challenges, and motivated by the fact that the optimal transmission strategy, in the sense of minimizing symbol error-rate (SER), in a point-to-point MIMO link with imperfect CSI is the combination of orthogonal spacetime block codes (OSTBC) and beamforming [6], we envisage a novel two-hop relay system in which the source and relay use OSTBCs along with their precoders. Due to the orthogonal properties of OSTBC, the joint optimization of the robust source and relay precoders is simplified without loss in the SNR optimality, and spectral efficiency for a class of full-rate OSTBC like the Alamouti code. Considering that the CSI of the S-R and R-D channels is imperfectly known at the source and relay nodes, respectively, we propose a robust design of source and relay precoders based on the worst-case approach, namely, maximization of the minimum SNR under sum power constraint of the source and relay. It is shown that this precoder optimization problem can be exactly reformulated as a convex problem which can be reduced to a joint source-relay power allocation problem. Using an approximation based on the assumption of medium to high average SNRs of two-hop links, highly efficient closed-form solutions for the power allocation problem are obtained by solving its Karush-Kuhn-Tucker (KKT) conditions. The accuracy of this approximation is validated through numerical results which show that the proposed robust precoder design with OSTBCs outperforms robust beamforming (without OSTBCs) based on the conservative worst-case design [5].

It is important to remark that in employing OSTBCs at the source and relay nodes, the resulting worst-case optimization structurally resembles the robust precoder design problem for a single-user MIMO link in [8]. In this regard, our work, in essence, extends the work in [8] to a MIMO relay system. Nevertheless, this extension is a difficult task since our objective function is a nontrivial function of the worst-case SNRs of the S-R and R-D channels, whereas in [8], the objective function is simply the worst-case SNR.

\section{Notations}

We use standard notational conventions as in [5]. 


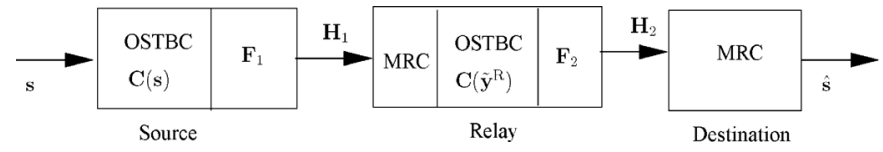

Fig. 1. Precoding of OSTBC for MIMO relay system.

\section{SySTEM MOdEL}

As shown in Fig. 1, the considered two-hop relay system consists of source, relay and destination nodes with $M_{1}, M_{2}$ and $M_{3}$ antennas, respectively. The direct link between the source and destination does not exist since it lies out of the coverage area of the source. The end-to-end signal transmission from the source to the destination takes place in two phases. In the first phase of $T \mathrm{~S}-\mathrm{R}$ channel uses, the relay receives $\mathbf{Y}_{\mathrm{r}}=$ $\mathbf{H}_{1} \mathbf{F}_{1} \mathbf{C}(\mathbf{s})+\mathbf{N}^{r}$, where $\mathbf{C}(\mathbf{s}) \in \mathcal{C}^{M_{1} \times T}\left(\mathbf{C}(\mathbf{s}) \mathbf{C}^{H}(\mathbf{s})=\right.$ $\left.\tilde{a}\|\mathbf{s}\|^{2} \mathbf{I}_{M_{1}}\right)$ is the OSTBC matrix formed from zero-mean complex source symbol $\mathbf{s}=\left[s_{1}, \ldots, s_{K}\right]$ with $\mathrm{E}\left\{\left|s_{k}\right|^{2}\right\}=1, \tilde{a}$ is code specific constant (e.g., $\tilde{a}=1$ for the Alamouti code [7]), $\mathbf{F}_{1} \in \mathcal{C}^{M_{1} \times M_{1}}$ is the source precoder, $\mathbf{H}_{1} \in \mathcal{C}^{M_{2} \times M_{1}}$ is the S-R MIMO channel and $\mathbf{N}^{r}$ is a matrix of the additive white Gaussian noise (AWGN) samples at the relay having zero mean and variance $\tilde{\sigma}_{1}^{2}$. The relay processes $\mathbf{Y}_{\mathrm{r}}$ with maximum ratio combiner (MRC). In this case, the S-R MIMO channel turns into $K$ parallel single-input single-output (SISO) channels. Thus, the signal received by the relay on the $k$ th S-R SISO channel can be given by [7]

$$
y_{k}^{\mathrm{R}}=\left\|\mathbf{H}_{1} \mathbf{F}_{1}\right\| s_{k}+n_{1, k}, k \in\{1, \ldots, K\}
$$

where $n_{1, k} \sim \mathcal{N}_{C}\left(0, \sigma_{1}^{2}=\tilde{\sigma}_{1}^{2} / \tilde{a}\right)$ is the AWGN at the relay for the $k$ th S-R SISO channel and follows complex Gaussian distribution with zero-mean and variance $\sigma_{1}^{2}$. The relay normalizes $\left\{y_{k}^{\mathrm{R}}\right\}_{k=1}^{K}$ yielding

$$
\tilde{y}_{k}^{\mathrm{R}}=\frac{y_{k}^{\mathrm{R}}}{\sqrt{\mathrm{E}\left\{\left|y_{k}^{\mathrm{R}}\right|^{2}\right\}}}=\frac{\left\|\mathbf{H}_{1} \mathbf{F}_{1}\right\| s_{k}+n_{1, k}}{\sqrt{\left\|\mathbf{H}_{1} \mathbf{F}_{1}\right\|^{2}+\sigma_{1}^{2}}} .
$$

In the second phase of $T$ R-D channel uses, the destination receives $\mathbf{Y}_{\mathrm{d}}=\mathbf{H}_{2} \mathbf{F}_{2} \mathbf{C}\left(\tilde{\mathbf{y}}^{\mathrm{R}}\right)+\mathbf{N}^{\mathrm{d}}$ where $\mathbf{C}\left(\tilde{\mathbf{y}}^{\mathrm{R}}\right) \in \mathcal{C}^{M_{2} \times T}$ is the OSTBC formed from $\tilde{\mathbf{y}}^{\mathrm{R}}=\left[\tilde{y}_{1}^{\mathrm{R}}, \ldots, \tilde{y}_{K}^{\mathrm{R}}\right], \mathbf{F}_{2} \in \mathcal{C}^{M_{2} \times M_{2}}$ is the relay precoder, $\mathbf{H}_{2} \in \mathcal{C}^{M_{3} \times M_{2}}$ is the R-D MIMO channel and $\mathbf{N}^{\mathrm{d}}$ is the matrix of AWGN samples at the destination having zero mean and variance $\tilde{\sigma}_{2}^{2}$.

As in the case of $k$ th S-R SISO channel, the signal received by the destination on the $k$ th R-D SISO channel becomes

$$
y_{k}^{D}=\left\|\mathbf{H}_{2} \mathbf{F}_{2}\right\| \tilde{y}_{k}^{\mathrm{R}}+n_{2, k}, \forall k
$$

where $n_{2, k} \sim \mathcal{N}_{C}\left(0, \sigma_{2}^{2}=\tilde{\sigma}_{2}^{2} / \tilde{a}\right)$ is the AWGN at the destination for the $k$ th R-D SISO channel. Using (2), (3) can be written as

$$
y_{k}^{D}=\frac{\left\|\mathbf{H}_{2} \mathbf{F}_{2}\right\|\left\|\mathbf{H}_{1} \mathbf{F}_{1}\right\| s_{k}+\left\|\mathbf{H}_{2} \mathbf{F}_{2}\right\| n_{1, k}}{\sqrt{\left\|\mathbf{H}_{1} \mathbf{F}_{1}\right\|^{2}+\sigma_{1}^{2}}}+n_{2, k} .
$$

The SNR at the destination can be expressed as

$$
\gamma=\frac{\left\|\mathbf{H}_{2} \mathbf{F}_{2}\right\|^{2}\left\|\mathbf{H}_{1} \mathbf{F}_{1}\right\|^{2}}{\left\|\mathbf{H}_{2} \mathbf{F}_{2}\right\|^{2} \sigma_{1}^{2}+\left\|\mathbf{H}_{1} \mathbf{F}_{1}\right\|^{2} \sigma_{2}^{2}+\sigma_{1}^{2} \sigma_{2}^{2}}=\frac{\gamma_{1} \gamma_{2}}{\gamma_{1}+\gamma_{2}+1}
$$

where $\gamma_{i}=\left\|\mathbf{H}_{i} \mathbf{F}_{i}\right\|^{2} / \sigma_{i}^{2}, i=1,2$. The transmit powers of the source and relay per OSTBC block can be given by $P_{1}=$
$K \tilde{a} \operatorname{tr}\left(\mathbf{F}_{1} \mathbf{F}_{1}^{H}\right)$ and $P_{2}=K \tilde{a} \operatorname{tr}\left(\mathbf{F}_{2} \mathbf{F}_{2}^{H}\right)$, respectively. The SNR expression (5) reduces to that of [10] when $\mathbf{F}_{i}=\mathbf{I}_{M_{i}}, \forall i$. Further, (5) appears to be similar in structure to the upper bound of the original SNR for a beamforming case without OSTBCs. This is evident from [11, eqs. (4) and (12)]. An obvious difference is that (5) is a function of source and relay precoders, whereas [11, eq. (12)] is the function of source and destination beamformers. Assuming perfect CSI, it is shown in [11] that there exists a relay matrix which achieves SNR upper bound ([11, eq. (12)]). As such, in perfect CSI case, the optimal value of $\gamma$ obtained with the optimal $\mathbf{F}_{i}$ will be the same as the optimal SNR obtained with the optimal source, relay and destination beamformers of [11]. This reveals that the proposed precoding with OSTBC does not incur any loss in SNR optimality compared to beamforming without OSTBC. On the other hand, if the source and relay have imperfect transmit side CSI, [11, eq. (12)] is neither achievable nor applicable. In this case, a new formulation of (5) is required and hence, the corresponding optimization problem cannot be addressed by the work in [11].

\section{PRECODER OPTIMIZATION}

We consider a time-division duplex system in which the transmit nodes estimate their channels using limited training/control signals received in reverse links, and use them as estimates in the forward links, due to channel reciprocity. Thus, the actual S-R and R-D channels can be modeled as $\mathbf{H}_{i}=\hat{\mathbf{H}}_{i}+\mathbf{E}_{i}, i=1,2$, where $\mathbf{E}_{i}$ is the uncertainty corresponding to the nominal (estimated) channel $\hat{\mathbf{H}}_{i}$. Although it is possible to consider that $\mathbf{E}_{i}$ are Gaussian distributed and lie in an ellipsoidal region with a certain probability, for simplicity and making model suitable for general sources of errors, as in [3], [8], we consider spherical region for $\mathbf{E}_{i}$ with unknown distribution. However, the upper bound for its Frobenius norm, $\delta_{i}$ is known. It is also possible to consider that the spectral norm of the error matrix is upper bounded by a known constant as in [9]. The problem of maximizing the minimum SNR can be written as

$$
\begin{array}{ll} 
& \max _{\mathbf{F}_{1}, \mathbf{F}_{2}\left\{\left\|\mathbf{E}_{i}\right\| \leq \delta_{i}\right\}_{i=1}^{2}} \gamma^{\mathrm{A}} \\
\text { s.t. } & \left\|\mathbf{F}_{1}\right\|^{2}+\left\|\mathbf{F}_{2}\right\|^{2} \leq P_{\mathrm{T}}
\end{array}
$$

where $P_{\mathrm{T}}$ is the total power budget (normalized by $K \tilde{a}$ ) and assumed to be smaller than the maximum power each node can transmit (e.g., in energy-constrained network), and

$$
\gamma^{\mathrm{A}}=\left[\sum_{i=1}^{2} \frac{\sigma_{i}^{2}}{\left\|\left(\hat{\mathbf{H}}_{i}+\mathbf{E}_{i}\right) \mathbf{F}_{i}\right\|^{2}}+\prod_{i=1}^{2} \frac{\sigma_{i}^{2}}{\left\|\left(\hat{\mathbf{H}}_{i}+\mathbf{E}_{i}\right) \mathbf{F}_{i}\right\|^{2}}\right]^{-1},
$$

is obtained by substituting $\mathbf{H}_{i}=\hat{\mathbf{H}}_{i}+\mathbf{E}_{i}$ into (5). From (7), we can see that $\gamma^{\mathrm{A}}$ increases monotonically with $\left\|\left(\hat{\mathbf{H}}_{i}+\mathbf{E}_{i}\right) \mathbf{F}_{i}\right\|^{2}, \forall i$, i.e., for given $\mathbf{F}_{1}$ and $\mathbf{F}_{2}$, the norm-bounded $\left\{\mathbf{E}_{i}\right\}_{i=1}^{2}$ that minimize $\left\|\left(\hat{\mathbf{H}}_{i}+\mathbf{E}_{i}\right) \mathbf{F}_{i}\right\|^{2}, \forall i$, also minimize $\gamma^{\mathrm{A}}$. Thus, (6) is equivalent to

$$
\begin{aligned}
& \max _{\left\{t_{i}, \mathbf{F}_{i}\right\}_{i=1}^{2}} f_{0} \triangleq\left[\frac{\sigma_{1}^{2}}{t_{1}}+\frac{\sigma_{2}^{2}}{t_{2}}+\frac{\sigma_{1}^{2}}{t_{1}} \frac{\sigma_{2}^{2}}{t_{2}}\right]^{-1} \\
& \text { s.t. } \min _{\left\{\left\|\mathbf{E}_{i}\right\| \leq \delta_{i}\right\}_{i=1}^{2}}\left\|\left(\hat{\mathbf{H}}_{i}+\mathbf{E}_{i}\right) \mathbf{F}_{i}\right\|^{2} \geq t_{i}, \forall i,(6 b) .
\end{aligned}
$$


For given $\left\{\mathbf{F}_{i}\right\}_{i=1}^{2}$, the inner optimization $\mathcal{P}_{i}: f_{i, \mathrm{wc}}\left(\mathbf{F}_{i}\right) \triangleq$ min $\left\|\left(\hat{\mathbf{H}}_{i}+\mathbf{E}_{i}\right) \mathbf{F}_{i}\right\|^{2}$ can be solved separately for each $\left\|\mathbf{E}_{i}\right\| \leq \delta_{i}$

$i$. At this point, it is important to explicitly highlight the difference between (8) and the problem solved in [8], which is $\mathcal{P}^{M}: \max _{\{\mathbf{F}\}} f_{\mathrm{wc}}(\mathbf{F})$ s.t. $\operatorname{tr}\left(\mathbf{F} \mathbf{F}^{H}\right) \leq P$, where the index $i$ is removed from $\mathbf{F}_{i}, f_{i, \mathrm{wc}}\left(\mathbf{F}_{i}\right)$ and $P_{i}$ to indicate that there is only one precoder (source). It is clear that $\mathcal{P}^{M}$ is only a special case of (8) when its objective function reduces to $t_{1}$ or $t_{2}$ and the number of precoders reduces to one. The similarity between (8) and $\mathcal{P}^{M}$ lies in the fact that the approach used for convex reformulation of $\mathcal{P}^{M}$ in [8] is applicable for reformulating (8b). However, in the underlying problem, we still must tackle with (8a) and obtain appropriate solutions for (8). These solutions require new derivations and are pursued both numerically and analytically, and validated in Section IV.

Since $\mathcal{P}_{i}$ is convex and Slater's condition holds, the duality gap between $\mathcal{P}_{i}$ and its dual problem is zero [12]. Hence, $\mathcal{P}_{i}$ can be replaced by its dual maximization problem $\mathcal{P}_{i}^{D}: \max _{\mu_{i} \geq 0} g_{i}\left(\mu_{i}\right)$, where $g_{i}\left(\mu_{i}\right)=\min _{\mathbf{E}_{i}} L\left(\mathbf{E}_{i}, \mu_{i}\right), L\left(\mathbf{E}_{i}, \mu_{i}\right)$ is the Lagrangian function for $\mathcal{P}_{i}$ and $\mu_{i}$ is the corresponding Lagrangian multiplier. Further, as discussed before, $f_{0}$ monotonically increases with $t_{i}$ where $t_{i}=\min _{\left\|\mathbf{E}_{i}\right\| \leq \delta_{i}}\left\|\left(\hat{\mathbf{H}}_{i}+\mathbf{E}_{i}\right) \mathbf{F}_{i}\right\|^{2}$ at the optimality. Thus, (8) can be expressed equivalently as

$$
\begin{aligned}
\min _{\left\{t_{i}, \mathbf{W}_{i}, \mu_{i}\right\}_{i=1}^{2}} & f_{0}^{-1} \text { s.t. } g_{i}\left(\mu_{i}\right) \geq t_{i}, \forall i, \\
& \operatorname{tr}\left(\mathbf{W}_{1}+\mathbf{W}_{2}\right) \leq P_{\mathrm{T}}
\end{aligned}
$$

where $\mathbf{W}_{i}=\mathbf{F}_{i} \mathbf{F}_{i}^{H} \succeq 0$. Solving $\min L\left(\mathbf{E}_{i}, \mu_{i}\right)$ [8], we can get $g_{i}\left(\mu_{i}\right)=\hat{\mathbf{h}}_{i}^{H} \mathbf{Z}_{i} \hat{\mathbf{h}}_{i}-\operatorname{vec}\left(\hat{\mathbf{H}}_{i} \mathbf{W}_{i}\right)^{H} \tilde{\mathbf{Z}}_{i} \operatorname{vec}\left(\hat{\mathbf{H}}_{i} \mathbf{W}_{i}\right)-\mu_{i} \delta_{i}^{2}$ where $\mathbf{Z}_{i}=\mathbf{W}_{i}^{T} \otimes \mathbf{I}_{M_{i+1}}, \tilde{\mathbf{Z}}_{i}=\tilde{\mathbf{W}}_{i}^{-1} \otimes \mathbf{I}_{M_{i+1}}, \tilde{\mathbf{W}}_{i}=\left(\mu_{i} \mathbf{I}_{M_{i}}+\right.$ $\left.\mathbf{W}_{i}^{T}\right)$ and $\hat{\mathbf{h}}_{i}=\operatorname{vec}\left(\hat{\mathbf{H}}_{i}\right)$. Then, using the Schur-Complement theorem [12], $g_{i}\left(\mu_{i}\right) \geq t_{i}$ can be expressed as the following linear matrix inequality

$$
\left[\begin{array}{cc}
\tilde{\mathbf{W}}_{i} \otimes \mathbf{I}_{M_{i+1}} & \operatorname{vec}\left(\hat{\mathbf{H}}_{i} \mathbf{W}_{i}\right) \\
\operatorname{vec}\left(\hat{\mathbf{H}}_{i} \mathbf{W}_{i}\right)^{H} & \hat{\mathbf{h}}_{i}^{H} \mathbf{Z}_{i} \hat{\mathbf{h}}_{i}-\mu_{i} \delta_{i}^{2}-t_{i}
\end{array}\right] \succeq 0 .
$$

Recall that in (9), we have $f_{0}^{-1}=\sigma_{1}^{2} t_{1}^{-1}+\sigma_{2}^{2} t_{2}^{-1}+\sigma_{1}^{2} \sigma_{2}^{2} t_{1}^{-1} t_{2}^{-1}$. Since $t_{1}$ and $t_{2}$ are positive scalars, both $t_{1}^{-1}$ and $t_{2}^{-1}$ are convex functions [12] of $t_{1}$ and $t_{2}$. Further, it can be shown that the Hessian matrix (the derivations are skipped due to space constraints) of $t_{1}^{-1} t_{2}^{-1}$ is positive semidefinite, i.e., $t_{1}^{-1} t_{2}^{-1}$ is also convex in $t_{1}$ and $t_{2}$. Consequently, the objective function of (9) is convex. It is still not straightforward to solve (9). Using an auxiliary variable $\tau$, we re-express (9) as

$$
\begin{gathered}
\min _{\left\{\tau, t_{i}, \mathbf{W}_{i}, \mu_{i}\right\}_{i=1}^{2}} f_{0}^{-1} \triangleq \sigma_{1}^{2} t_{1}^{-1}+\sigma_{2}^{2} t_{2}^{-1}+\sigma_{1}^{2} \sigma_{2}^{2} \tau^{-2} \text { s.t. } \\
\tau^{2} \leq t_{1} t_{2},(10),(9 b) .
\end{gathered}
$$

Since hyperbolic constraint $\tau^{2} \leq t_{1} t_{2}$ can be expressed as the convex constraint $\left\|\left[\sqrt{2} \tau, t_{1}, t_{2}\right]\right\| \leq t_{1}+t_{2}$, we get

$$
\begin{array}{r}
\min _{\left\{\tau, t_{i}, \mathbf{W}_{i}, \mu_{i}\right\}_{i=1}^{2}} f_{0}^{-1} \text { s.t. }(10),(9 b), \\
t_{1}+t_{2} \geq\left\|\left[\sqrt{2} \tau, t_{1}, t_{2}\right]\right\|
\end{array}
$$

which can be solved using interior-point methods [12] with a complexity of $\mathcal{O}\left(M^{9}\right)$ for $M_{i}=M_{i+1}=M$. We now provide an approximate semi-analytical approach which solves (12) with a complexity of $\mathcal{O}\left(M^{3}\right)$, same as performing an eigen-decomposition (ED) of an $M \times M$ matrix.

1) Closed-Form Solution: Let the EDs of $\mathbf{W}_{i}$ and $\hat{\mathbf{H}}_{i}^{H} \hat{\mathbf{H}}_{i}$ be given by $\mathbf{W}_{i}=\mathbf{U}_{i} \boldsymbol{\Lambda}_{\mathbf{W}_{i}} \mathbf{U}_{i}^{H}$ and $\hat{\mathbf{H}}_{i}^{H} \hat{\mathbf{H}}_{i}=\mathbf{U}_{\hat{\mathbf{H}}_{i}} \boldsymbol{\Lambda}_{\hat{\mathbf{H}}_{i}} \mathbf{U}_{\hat{\mathbf{H}}_{i}}^{H}$ with their respective eigenvalues $\lambda_{k}^{\mathbf{W}_{i}}$ and $\lambda_{k}^{\hat{\mathbf{H}}_{i}}, k=1, \ldots, M_{i}$, in the non-decreasing order. Note that $\lambda_{k}^{\hat{\mathbf{H}}_{i}}=0$ for all $k>r_{i} \triangleq$ $\operatorname{rank}\left(\hat{\mathbf{H}}_{i}\right)$. Using these EDs in $g_{i}\left(\mu_{i}\right)$ and applying Lemma 3 of [8], it can be easily shown that $\mathbf{U}_{i}=\mathbf{U}_{\hat{\mathbf{H}}_{i}}, \forall i$, are optimal for (9). Let us define $\tilde{g}_{i}\left(\mu_{i}\right)$ as

$$
\tilde{g}_{i}\left(\mu_{i}\right) \triangleq g_{i}\left(\mu_{i}, \mathbf{U}_{i}=\mathbf{U}_{\hat{\mathbf{H}}_{i}}\right)=\sum_{k=1}^{r_{i}} \frac{\lambda_{k}^{\mathbf{W}_{i}} \lambda_{k}^{\hat{\mathbf{H}}_{i}} \mu_{i}}{\lambda_{k}^{\mathbf{W}_{i}}+\mu_{i}}-\mu_{i} \delta_{i}^{2} .
$$

Since no power will be allocated to zero $\lambda_{k}^{\hat{\mathbf{H}}_{i}}$, we can consider $\lambda_{k}^{\mathbf{W}_{i}}=0$ for $k>r_{i}$ and obtain the total power as $\tilde{p}=\sum_{k=1}^{r_{1}} \lambda_{k}^{\mathbf{W}_{1}}+\sum_{j=1}^{r_{2}} \lambda_{j}^{\mathbf{W}_{2}}$. Then, using the approximation $t_{1} / \sigma_{1}^{2}+t_{2} / \sigma_{2}^{2} \gg 1$, (9) can be written as

$$
\min _{\left\{t_{i}, \mu_{i}, \lambda_{k}^{\mathbf{w}_{i}}\right\}_{i=1}^{2}} \tilde{f}_{0} \text { s.t. } \tilde{g}_{i}\left(\mu_{i}\right) \geq t_{i}, \forall i, \tilde{p} \leq P_{\mathrm{T}}
$$

where $\tilde{f}_{0}=\sigma_{1}^{2} t_{1}^{-1}+\sigma_{2}^{2} t_{2}^{-1}$. Because $\tilde{f}_{0}$ is convex, $\tilde{g}_{i}\left(\mu_{i}\right)$ can be proved to be a concave function of $\mu_{i}$ and $\lambda_{k}^{\mathbf{W}_{i}}$ [8], and Slater's condition holds and is sufficient for constraint qualification, (13) can be solved by using the KKT conditions. The Lagrangian function for (13) is given by

$\tilde{L}\left(t_{i}, \mu_{i}, \lambda_{k}^{\mathbf{W}_{i}}, \nu_{i}, \nu\right)=\tilde{f}_{0}+\sum_{i=1}^{2} \nu_{i}\left(t_{i}-\tilde{g}_{i}\left(\mu_{i}\right)\right)+\nu\left(\tilde{p}-P_{\mathrm{T}}\right)$

where $\nu_{i} \geq 0$ and $\nu \geq 0$ are the Lagrangian multipliers.

Proposition: The KKT optimality conditions for (13) results into the optimal objective function given by

$$
\tilde{f}_{0}^{-1}=\frac{P_{\mathrm{T}}\left(c_{1}-\tilde{\alpha}_{1} \sqrt{\frac{\nu}{\nu_{1}}}\right)\left(c_{2}-\tilde{\alpha}_{2} \sqrt{\frac{\nu}{\nu_{2}}}\right)}{\tilde{s}\left[\sigma_{2} \sqrt{\frac{\nu_{2}}{\nu}}+\sigma_{1} \sqrt{\frac{\nu_{1}}{\nu}}\right]}
$$

where $\tilde{\alpha}_{i}=\sum_{k=1}^{n^{i}} \sqrt{\lambda_{k}^{\hat{\mathbf{H}}_{i}}}, c_{i}=\bar{\alpha}_{i}-\delta_{i}^{2}, \bar{\alpha}_{i}=\sum_{k=1}^{n^{i}} \lambda_{k}^{\hat{\mathbf{H}}_{i}}$, $n^{i} \in\left\{1, \ldots, r_{i}\right\}$ is an integer such that $\lambda_{n^{i}+1}^{\hat{\mathbf{H}}_{i}} \leq \nu / \nu_{i}<\lambda_{n^{i}}^{\hat{\mathbf{H}}_{i}}$, $\tilde{s}$ as a function of $\left\{\nu / \nu_{i}, n^{i}\right\}$ is first defined in Appendix.

Proof: Please see Appendix.

\section{NumericAl RESUlts}

We compare the worst-case performance of robust precoding schemes with the OSTBC, namely, the proposed method $(\mathrm{PM})$, beamforming $(\mathrm{BF}+\mathrm{OSTBC})$ and equal power allocation (EP+OSTBC). We also show the performance of the robust beamforming without OSTBC (RBF). For the latter three methods, the worst-case objective functions as a function of power allocation parameters are obtained using the worst-case errors in [5]. For BF+OSTBC, we get $\mathbf{F}_{i}=\left[\sqrt{b_{i}} \mathbf{u}_{\hat{\mathbf{H}}_{i}}^{1}, \mathbf{0}, \ldots, \mathbf{0}\right]$, where $\mathbf{u}_{\hat{\mathbf{H}}_{i}}^{1}$ is the first column of $\mathbf{U}_{\hat{\mathbf{H}}_{i}}$. For EP+OSTBC, we have $\mathbf{F}_{i}=\sqrt{b_{i}} \mathbf{I}_{M_{i}}$. Then, we solve the problem

$$
\max _{b_{1}, b_{2}} \frac{b_{1} \bar{\gamma}_{1} b_{2} \bar{\gamma}_{2}}{b_{1} \bar{\gamma}_{1}+b_{2} \bar{\gamma}_{2}+1} \text {, s.t. } b_{1}+b_{2} \leq P_{\mathrm{T}}
$$




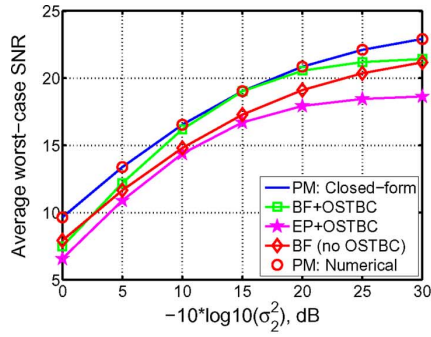

(a)

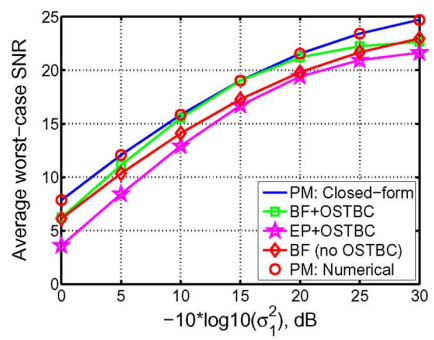

(b)
Fig. 2. Performance comparison between different robust schemes. (a) Worstcase SNR versus $\sigma_{2}^{2}$. (b) Worst-case SNR versus $\sigma_{1}^{2}$.

where $\bar{\gamma}_{i}=\sigma_{i}^{-2}\left(\tilde{\lambda}_{1, i}-\delta_{i}\right)^{2}, \tilde{\lambda}_{1, i} \triangleq \sqrt{\lambda_{1}^{\hat{\mathbf{H}}_{i}}}$, for BF+OSTBC, and $\bar{\gamma}_{i}=\sigma_{i}^{-2}\left(\left\|\hat{\mathbf{H}}_{i}\right\|-\delta_{i}\right)^{2}$ for EP+OSTBC with $b_{i}=$ $0.5 P_{\mathrm{T}} / M_{i}$. In the RBF case, the source, relay and destination beamformers form the matching solution as in [11] for the worst-case errors [5]. The source and relay powers in this case are optimized by solving

$$
\max _{a, b} \frac{a b x}{b y+\sigma_{2}^{2}} \text {, s.t. } a+b\left(a\left(\tilde{\lambda}_{1,1}+\delta_{1}\right)^{2}+\sigma_{1}^{2}\right) \leq P_{\mathrm{T}}
$$

where $x=\left(\tilde{\lambda}_{1,1}-\delta_{1}\right)^{2}\left(\tilde{\lambda}_{1,2}-\delta_{2}\right)^{2}$ and $y=\left(\tilde{\lambda}_{1,2}+\delta_{2}\right)^{2}$. Simulation results are obtained by averaging over 200 independent realizations of $\hat{\mathbf{H}}_{1}$ and $\hat{\mathbf{H}}_{2}$ whose entries are i.i.d. zero-mean complex Gaussian random variables with variance $\sqrt{2}$. In all simulations, we take $M_{1}=M_{2}=2$ (Alamouti code with $\tilde{a}=1), M_{3}=4, P_{\mathrm{T}}=2, \delta_{i}=d_{i}\left\|\hat{\mathbf{H}}_{i}\right\|_{2}$ with $d_{1}=d_{2}=0.1$. The average worst-case SNRs (in $\mathrm{dB}$ ) versus $\sigma_{2}^{2}$ with $\sigma_{1}^{2}=$ 0.0316 and $\sigma_{1}^{2}$ with $\sigma_{2}^{2}=0.0316$, are respectively, shown in Fig. 2(a) and (b) for different schemes. These figures show that there exists negligible difference between the exact numerical and approximate semi-analytical solutions of the PM. The PM outperforms all other designs. The respective gains of the PM over $\mathrm{BF}+\mathrm{OSTBC}, \mathrm{RBF}$, and $\mathrm{EP}+\mathrm{OSTBC}$ are up to $1.5 \mathrm{~dB}, 2 \mathrm{~dB}$, and $4 \mathrm{~dB}$, which is significant since the PM (semi-analytical) can be solved with the similar minimal degree of complexity as the other three methods.

\section{APPENDIX}

Notice that $\tilde{g}_{i}\left(\mu_{i}\right)=t_{i}, \forall i$ hold at the optimality of (9). It can be also verified by contradiction that at the optimality, $\tilde{p} \leq P_{\mathrm{T}}$ in (13) becomes equality. The related discussions are skipped due to limited space. Solving the partial derivatives of $\tilde{L}$ w.r.t. $t_{1}$ and $t_{2}$, we obtain $\nu_{1}=\sigma_{1}^{2} t_{1}^{-2}, \nu_{2}=\sigma_{2}^{2} t_{2}^{-2}$, which shows that $\nu_{i}=0$ is not possible due to the facts that $\sigma_{i}^{2} \neq 0$ and $t_{i}$ is finite (cf. (8)). Solving the partial derivative of $\tilde{L}$ w.r.t. $\lambda_{k}^{\mathbf{W}_{i}}$, we get $\nu_{i}\left(\lambda_{k}^{\hat{\mathbf{H}}_{i}} \mu_{i}^{2} /\left(\lambda_{k}^{\mathbf{W}_{i}}+\mu_{i}\right)^{2}\right)=\nu$. Since $\nu_{i} \neq 0, \mu_{i}=0$ cannot be true at the optimality, it is clear that $\nu \neq 0$. Hence, we get

$$
\lambda_{k}^{\mathbf{W}_{i}}= \begin{cases}\left(\sqrt{\frac{\lambda_{k}^{\hat{\mathbf{H}}_{i}} \nu_{i}}{\nu}}-1\right) \mu_{i}, & \text { for } \frac{\nu}{\nu_{i}}<\lambda_{k}^{\hat{\mathbf{H}}_{i}}, \\ 0, & \text { for } \frac{\nu}{\nu_{i}} \geq \lambda_{k}^{\hat{\mathbf{H}}_{i}},\end{cases}
$$

$\forall i, k$. Note that (17) corresponds to water-filling type of solution. In contrast to the single-hop MIMO link [3], (17) consists of two stage water-filling, i.e., one at the source and the other at the relay where the corresponding water-levels are associated through the common parameter $\nu$. Substituting (17) into the complementary slackness condition $\nu_{i}\left(t_{i}-\tilde{g}_{i}\left(\mu_{i}\right)\right)=0$, we obtain

$$
t_{i}=\mu_{i}\left(\bar{\alpha}_{i}-\tilde{\alpha}_{i} s_{i}-\delta_{i}^{2}\right), \forall i
$$

where $\bar{\alpha}_{i}=\sum_{k=1}^{n^{i}} \lambda_{k}^{\hat{\mathbf{H}}_{i}}, \tilde{\alpha}_{i}=\sum_{k=1}^{n^{i}} \sqrt{\lambda_{k}^{\hat{\mathbf{H}}_{i}}}, s_{i}=\sqrt{\nu / \nu_{i}}$, and $n^{i}$ is the integer such that $\lambda_{k}^{\mathbf{W}_{i}}$ in (17) are $\lambda_{k}^{\mathbf{W}_{i}}=0$ for all $k$ where $k>n^{i}$. Applying (17) into the KKT condition $\nu\left(\tilde{p}-P_{\mathrm{T}}\right)=0$, we get

$$
\mu_{1}\left(\tilde{\alpha}_{1} s_{1}^{-1}-n^{1}\right)+\mu_{2}\left(\tilde{\alpha}_{2} s_{2}^{-1}-n^{2}\right)=P_{\mathrm{T}} .
$$

Some manipulations of (18) and $\nu_{i}=\sigma_{i}^{2} t_{i}^{-2}, \forall i$, gives

$$
\frac{t_{2}}{t_{1}}=\frac{\sigma_{2}}{\sigma_{1}} \sqrt{\frac{\nu_{1}}{\nu_{2}}}=\frac{\mu_{2}\left(c_{2}-s_{2} \tilde{\alpha}_{2}\right)}{\mu_{1}\left(c_{1}-s_{1} \tilde{\alpha}_{1}\right)}
$$

where $c_{i}=\bar{\alpha}_{i}-\delta_{i}^{2}(i=1,2)$. Solving (20) and (19), we get

$$
\mu_{1}=\frac{P_{\mathrm{T}} s_{1} \sigma_{1}\left(c_{2}-\tilde{\alpha}_{2} s_{2}\right)}{\tilde{s}}, \mu_{2}=\frac{P_{\mathrm{T}} s_{2} \sigma_{2}\left(c_{1}-\tilde{\alpha}_{1} s_{1}\right)}{\tilde{s}},
$$

where $\tilde{s}=\left(\tilde{\alpha}_{1}-n^{1} s_{1}\right)\left(c_{2}-\tilde{\alpha}_{2} s_{2}\right) \sigma_{1}+\left(\tilde{\alpha}_{2}-n^{2} s_{2}\right)\left(c_{1}-\right.$ $\left.\tilde{\alpha}_{1} s_{1}\right) \sigma_{2}$. The remaining task is to determine $\nu / \nu_{i}$ and $n^{i}$. Analyzing (17), it is easy to verify $\lambda_{n^{i}+1}^{\hat{\mathbf{H}}_{i}} \leq \nu / \nu_{i}<\lambda_{n^{i}}^{\hat{\mathbf{H}}_{i}}$. Solving $\delta \tilde{L} / \delta \mu_{i}=0$ and using (17), we can obtain $\nu / \nu_{i}$ as a function of $n^{i}$. Then, $\nu / \nu_{i}$ that satisfies $\lambda_{n^{i}+1}^{\hat{\mathbf{H}}_{i}} \leq \nu / \nu_{i}<\lambda_{n^{i}}^{\hat{\mathbf{H}}_{i}}$ is chosen for each $i$. Finally, using (18) and (21), we get (14). This completes the proof of the Proposition.

\section{REFERENCES}

[1] C. Xing, S. Ma, and Y.-C. Wu, "Robust joint design of linear precoder and destination equalizer for dual-hop amplify-and-forward MIMO relay systems," IEEE Trans. Signal Process., vol. 58, no. 4, pp. 2273-2283, Apr. 2010.

[2] H. W. Je, D. H. Kim, and K. B. Lee, "Joint precoding for MIMO-relay systems with partial channel state information," in Proc. IEEE ICC'09, Dresden, Germany, Jun. 2009.

[3] A. Abdel-Samad, T. N. Davidson, and A. B. Gershman, "Robust transmit eigen beamforming based on imperfect channel state information," IEEE Trans. Signal Process., vol. 54, no. 5, May 2006.

[4] E. A. Gharavol, Y.-C. Liang, and K. Mouthaan, "Robust linear beamforming for MIMO relay with imperfect channel state information," in Proc. IEEE PIMRC'10, Istanbul, Turkey, Sep. 2010.

[5] B. K. Chalise and L. Vandendorpe, "MIMO relay design for multipoint-to-multipoint communications with imperfect channel state information," IEEE Trans. Signal Process., vol. 57, no. 7, pp. 2785-2796, Jul. 2009 .

[6] S. Zhou and G. B. Giannakis, "Optimal transmitter eigen-beamforming and space-time block coding based on channel mean feedback," IEEE Trans. Signal Process., vol. 50, no. 10, pp. 2599-2613, Oct. 2002.

[7] A. Hjorugnes and D. Gesbert, "Precoding of orthogonal-space time block codes in arbitrarily correlated MIMO channels: Iterative and closed-form solutions," IEEE Trans. Wireless Commun., vol. 6, no. 3, pp. 1072-1082, Mar. 2007.

[8] J. Wang and D. P. Palomar, "Worst-case robust MIMO transmission with imperfect channel knowledge," IEEE Trans. Signal Process., vol. 57, no. 8, Aug. 2009.

[9] J. Wang and M. Payaro, "On the robustness of transmit beamforming," IEEE Trans. Signal Process., vol. 58, no. 11, pp. 5933-5938, Nov. 2010.

[10] L. Yang and Q. T. Zhang, "Performance analysis of MIMO relay wireless networks with orthogonal STBC," IEEE Trans. Veh. Technol., vol. 59, no. 7, pp. 3668-36674, Sep. 2010.

[11] B. Khoshnevis, W. Yu, and R. Adve, "Grassmannian beamforming for MIMO amplify-and-forward relaying," IEEE J. Sel. Areas Commun., vol. 26, no. 8, pp. 1397-1407, Oct. 2008.

[12] S. Boyd and L. Vandenberghe, Convex Optimization. Cambridge, U.K.: Cambridge Univ. Press, 2004. 